\title{
LOS ISÓPODOS TERRESTRES DE GALICIA, ESPAÑA (CRUSTACEA: ISOPODA, ONISCIDEA)
}

\author{
Julio Cifuentes \\ Departamento de Biología (Zoología), Facultad de Ciencias, Universidad Autónoma de Madrid, 28049 Cantoblanco, Madrid, \\ España. E-mail: jcifcol@gmail.com - ORCID-iD: https://orcid.org/0000-0002-9569-6973
}

\section{RESUMEN}

La revisión de la colección de Isopoda del Museo Nacional de Ciencias Naturales de Madrid (MNCN) y la del autor, ha permitido estudiar 431 ejemplares de 19 especies de isópodos terrestres de Galicia, España. Una especie se cita por la primera vez para Pontevedra y Galicia, Tylos europaeus Arcangeli, 1938, y Porcellio herminiensis (Vandel, 1946) se cita por primera vez para la provincia de A Coruña. La fauna conocida de isópodos terrestres de Galicia está formada por 38 especies, 28 de ellas se encuentran en la provincia de Pontevedra, 24 especies en A Coruña, 15 especies en Orense y 14 especies en Lugo.

Palabras clave: Crustáceos; isópodos terrestres; distribución; Galicia; España.

\section{ABSTRACT}

\section{The terrestrial isopods from Galicia, Spain (Crustacea: Isopoda, Oniscidea)}

The revision of the Isopoda collections belonging to the Museo Nacional de Ciencias Naturales de Madrid (MNCN) and to the author, allowed the study of 431 specimens ascribed to 19 species of the terrestrial isopods from Galicia, Spain. One species is cited for the first time from Pontevedra and Galicia, Tylos europaeus Arcangeli, 1938, and Porcellio herminiensis (Vandel, 1946) is cited for the first time from the province of A Coruña. The known terrestrial isopodological fauna of Galicia is formed by 38 species, 28 of them are found in the province of Pontevedra, 24 species in A Coruña, 15 species in Orense and 14 species in Lugo.

Key words: Crustacea; terrrestrial isopoda; distribution; Galicia; Spain.

Recibido/Received: 09/05/2019; Aceptado/Accepted: 31/07/2019; Publicado en línea/Published online: 16/09/2019

Cómo citar este artículo/Citation: Cifuentes, J. 2019. Los isópodos terrestres de Galicia, España (Crustacea: Isopoda, Oniscidea). Grael/sia, 75(2): e098. https://doi.org/10.3989/graellsia.2019.v75.243

Copyright: (C) 2019 SAM \& CSIC. This is an open-access article distributed under the terms of the Creative Commons Attribution 4.0 International (CC BY 4.0) License.

\section{Introducción}

La primera referencia bibliográfica a los isópodos terrestres gallegos se remonta a finales del s. XIX, cuando De Buen (1887) cita la especie Ligia oceanica (Linnaeus, 1767) de Ferrol (A Coruña) y de la ría de Marín (Pontevedra). Posteriormente Dollfus $(1892,1893,1897)$ eleva a 12 las especies conocidas de estos crustáceos en Galicia. Ya en los años 50 del siglo pasado, Schmölzer (1955a) añade otras 22, y posteriormente (Schmölzer, 1971) otras dos más. Finalmente, los trabajos de Vandel (1962), Bilton (1992, 1997), Vázquez Felechosa \& Anadón (2001), García (2005) y Gregory et al. (2012), completan con otras siete, 43 en total, las especies citadas hasta este trabajo. El presente trabajo tiene por objetivos contribuir al conocimiento de la fauna de isópodos de Galicia y de España, aportando nuevos datos sobre su distribución y actualizar el catálogo de los isópodos gallegos. 


\section{Material y métodos}

Se han estudiado 431 ejemplares pertenecientes a 19 especies de isópodos terrestres de la colección del Museo Nacional de Ciencias Naturales de Madrid (MNCN) y la del autor (JC). Para la determinación del material se han realizado preparaciones microscópicas con la resina sintética DMHF (dimetil hidantoína formaldehido) y se han fotografiado con una cámara digital Bresser MikroCam SP 3.1 montada en un microscopio URA 106 y en un estereo-microscopio URA Technic 237.

\section{Resultados}

Familia TYLIDAE Milne-Edwards, 1840

Género Tylos Audouin, 1826

Tylos europaeus Arcangeli, 1938

Material estudiado. Pontevedra: O Grove, 8-VIII-1953, 1 §ै, W. Steiner leg., MNCN 20.04/6109.

Citas del resto de España. Alicante (Schmölzer, 1971), Almería (Vandel, 1953), Barcelona (Soika, 1954), Islas Baleares (García \& Cruz, 1993, 1996), Málaga (Koch, 1856; Budde-Lund, 1885), Murcia (Soika, 1954) y Valencia (Soika, 1954).

Comentarios. Tylos europaeus es una especie halófila que coloniza el área supramareal. Si bien Vandel (1960: 107, fig. 48) sombrea toda la costa peninsular para indicar el área de distribución de esta especie, hasta el presente en España solamente se ha citado de las provincias indicadas, por lo que es nueva cita para Pontevedra, y Galicia. La cita más proxima es la de Vila Praia de Âncora en el distrito portugués de Viana do Castelo (Gregory et al., 2012).

Familia LIGIIDAE Brandt \& Ratzeburg, 1831

Género Ligia Fabricius, 1798

Ligia oceanica (Linnaeus, 1767)

Material estudiado. Pontevedra: La Guardia, 3-VIII1985, 2 q 9 y 5 inmaduros, JC leg. y col.; O Grove, 8-VIII-1953, 1 inmaduro, W. Steiner leg., MNCN 20.04/9689; Portonovo, 30-VII-1985, 2 q $ᄋ$ y 6 inmaduros, JC leg. y col.; Vigo, Bouzas, 13-IX-1956, 12 $\widehat{\jmath}$, 6 우 y 19 inmaduros, Eugenio Ortíz de Vega leg., MNCN 20.04/9917.

Citas de Galicia. A Coruña: Ferrol (De Buen, 1887). Ría del Burgo (Dollfus, 1892). Pontevedra: Marín, ría (De Buen, 1887). Oia (Gregory et al., 2012).

Citas del resto de España. Asturias (De Buen, 1887), Vizcaya (Budde-Lund, 1885; Dollfus, 1892).

Comentarios. A pesar de las escasas citas, esta especie debe de encontrarse en toda la costa atlántica peninsular, pero según Vandel (1960), no se encuentra en las costas mediterráneas, siendo la cita de Málaga (Budde-Lund, 1885) un error de determinación.
Familia TRICHONISCIDAE Sars, 1898

Género Haplophthalmus Schoebl, 1860

Haplophthalmus danicus Budde-Lund, 1880

Material estudiado. Pontevedra: O Grove, 6-VIII-1952, 1 ồ y 3 oq $q$, H. Franz leg., MNCN 20.04/9659.

Citas de Galicia. Orense: Beade (Gregory et al., 2012). Peña Trevinca (Schmölzer, 1955a, 1971). Pontevedra: A Carballeiro (Schmölzer, 1955a). Camposancos; Goján y Oia (Gregory et al., 2012). Pontevedra (Schmölzer, 1971).

CitAs del RESto DE EsPañA. Barcelona (Schmölzer, 1971; Cruz, 1991), Islas Baleares (Schmölzer, 1971; García \& Cruz, 1996; García, 2009), Sevilla (Cifuentes \& Tinaut, 2019) y Tarragona (Arcangeli, 1924; Cruz, 1991).

Comentarios. Es una especie expansiva que se encuentra en Europa, norte de África, Asia Menor y gran parte del norte de América (Vandel, 1960). En la península ibérica por el momento sus citas son muy dispersas, por lo que nuevos estudios podrán precisar mejor su área de distribución.

Género Miktoniscus Kesselyak, 1930

Miktoniscus bisetosus Vandel, 1946

Citas de Galicia. Pontevedra: Bayona (Cabo Silleiro) y Marín (Schmölzer, 1955a, 1971). Gondomar (Gregory et al., 2012).

Citas del resto de España. Asturias (Schmölzer, 1971).

Comentarios. Es una especie de distribución muy restringida, solamente se conoce de las provincias españolas de Asturias y Pontevedra, y del distrito portugués de Viana do Castelo (Vandel, 1946; Schmölzer, 1955a, 1971; Gregory et al., 2012).

\section{Miktoniscus patiencei Vandel, 1946}

Material estudiado. Pontevedra: Pontevedra, 9-VII-1952, 1 đ̃ y 3 우, H. Franz leg., col. Schmölzer, MNCN $20.04 / 9675$.

Citas de Galicia. Pontevedra: Bayona y Oia (Gregory et al., 2012).

Comentarios. Según Schmalfuss (2003), esta especie se encuentra en el norte de Francia, Escocia, Inglaterra, Irlanda y las islas del Canal de la Mancha, y también la menciona con dudas de Madeira. En la península ibérica solamente se ha citado de la provincia de Pontevedra.

Los ejemplares estudiados, con alguna pequeña diferencia, se ajustan a la descripción que hace Vandel de M. patiencei, pero la fecha de captura, 9-VII-1952, el colector H. Franz leg., y el pertenecer a la col. Schmölzer, induce a pensar que estos ejemplares (junto con otros no encontrados en el $\mathrm{MNCN}$ ) corresponden a las citas de Schmölzer (1955a, 1971) de M. bisetosus de Pontevedra, y es por tanto posible también que la cita de Asturias de M. bisetosus de Schmölzer (1971), corresponda en realidad a $M$. patiencei. 
Género Oritoniscus Racovitza, 1908

Oritoniscus simplex Vandel, 1957

Citas de Galicia. A Coruña: Cerdedo (Sierra oeste Ortigueira-A Barqueira) (Schmölzer, 1971). Santiago, bosque Cernadas (Schmölzer, 1955a, 1971). Lugo: Piornedo (Schmölzer, 1955a; Vandel, 1957, 1960). Pontevedra: Barra; Bayona, cabo Silleiro; Nigrán, monte Ferro; Vigo, ría (Schmölzer, 1971).

Citas del RESto de España. Asturias (Schmölzer, 1955a, 1971; Vandel, 1957; Vázquez Felechosa et al., 2004), Cantabria (Schmölzer, 1971), Guipúzcoa (Schmölzer, 1971), La Rioja (Schmölzer, 1971), León (Schmölzer, 1971) y Navarra (Vandel, 1957; Schmölzer, 1971).

Comentarios. La citas de O. flavus (Budde-Lund, 1906) de Santiago y Piornedo (Schmölzer, 1955a) corresponden en realidad a $O$. simplex, especie cuya área de distribución se extiende desde los pirineos occidentales hasta Galicia. O. flavus sin embargo es una especie pirenaica, citada de España de las provincias de Barcelona (Arcangeli, 1924; Cruz, 1991), Gerona (Arcangeli, 1924; Schmölzer, 1971; Cruz, 1991) y Lérida (Vandel, 1957; Dalens et al., 1996).

Género Trichoniscoides G. O. Sars, 1899

Trichoniscoides ancaresi Schmölzer, 1955

Material estudiado. Lugo, Cabana Vella (Ancares), 30-VII1952, 1 đ̊ y 1 ㅇ, H. Franz leg., MNCN 20.04/9667.

Citas de Galicia. Lugo: Sierra de Ancares, Cabana Vella y Piornedo (Schmölzer, 1955a, 1971). Cabana Vella (Cifuentes, 2019).

Citas del resto de España. Cantabria (Schmölzer, 1971).

Comentarios. Esta especie ha sido muy escasamente citada de algunas localidades del noroeste peninsular.

Trichoniscoides lusitanus Vandel, 1946

Material estudiado. Pontevedra: Cangas, Playa Castiñeira, 4-VII-1952, 1 q, H. Franz leg., MNCN 20.04/9657.

Citas de Galicia. A Coruña: Santiago, bosque de Cernadas (Schmölzer, 1971). Orense: Avión; Beade y Beiro (Gregory et al., 2012). Lobios, Puerto de la Home (Schmölzer, 1971). Pontevedra: Amorin; As Eiras y Gondomar (Gregory et al., 2012). Cangas, Playa Castiñeira (Cifuentes, 2019). Marín (Schmölzer, 1955a, 1971). Oia y Puerto de Moncelos (Gregory et al., 2012).

Citas del resto de España. Cantabria (Schmölzer, 1971).

Comentarios. Además de las citas anteriores, esta especie se conoce de tres distritos portugueses, Braga, Porto y Viana do Castelo (Vandel, 1946; Gregory et al., 2012).

Trichoniscoides modestus Racovitza, 1908

Citas de Galicia. Lugo: Samos, cueva de Santalla de Lozera (Schmölzer, 1955a).

Comentarios. Trichoniscoides modestus es una especie del sur de Francia, de la que solamente ha sido citada de España de la localidad ya señalada por Schmölzer (1955a), por lo que Schmalfus (2003) considera que esta cita debe de ser confirmada. Por lo tanto no puede considerarse como una especie de Galicia.

Género Trichoniscus Brandt, 1833

Trichoniscus provisorius Racovitza, 1908

Citas de Galicia. A Coruña: Pasaje (Dollfus, 1893). Orense: Avión y Beade (Gregory et al., 2012). Pontevedra: Amorin; As Eiras-Goján; Camposancos y Oia (Gregory et al., 2012).

Citas del resto de España. Alicante, Barcelona, Cádiz, Guipúzcoa, Huesca, Islas Baleares, Lérida, Málaga, Navarra y Sevilla (Arcangeli, 1924, 1935; Vandel, 1953, 1960, 1961; Schmölzer, 1971; Tabacaru, 1974; Cifuentes, 1984; Bellés et al., 1989; Cruz, 1989, 1991; García \& Cruz, 1996; García, 2009; González Silvestre, 2015).

Comentarios. Dado que esta especie y la siguiente se consideraban subespecies de T. pusillus, no es facil establecer en algunos casos si las citas se refieren a una $u$ otra de ellas. Es la especie de Trichoniscus con mayor área de distribución conocida en la península.

Trichoniscus pusillus Brandt, 1833

Material estudiado. A Coruña: Pasaje, 50 q $q$ (5 ovígeras), MNCN 20.04/7679.

Citas de Galicia. A Coruña: Pasaje (Dollfus, 1893). Orense: Avión, valle del río Valdeiras; Beade (Gregory et al., 2012). Pontevedra: Amorin; As Eiras-Goján; Camposancos y Oia (Gregory et al., 2012).

Citas del resto de España. Álava (Vivar et al., 1984), Barcelona (Arcangeli, 1924, 1925), Cuenca (Pollo Zorita, 1986a), Gerona (Arcangeli, 1924), Guadalajara (Pollo Zorita, 1986a), Madrid (Pollo Zorita, 1986a), Navarra (Cifuentes, 1984; Cruz, 1991) y Vizcaya (Vivar et al., 1984).

Comentarios. Los ejemplares que hemos estudiado, probablemente sean los que citó Dollfus (1893). Es una especie humícola que solamente puede vivir en zonas con alta humedad ambiental. A pesar de ello, es una especie expansiva de amplia distribución en Europa (Vandel, 1960). Los machos son muy raros, 1,6\% de media según Vandel (1960).

Familia ONISCIDAE Latreille, 1806

Género Oniscus Linnaeus, 1758

Oniscus ancarensis Bilton, 1992

Citas de Galicia. Lugo: Sierra de Ancares, Cabana Vella; Os Cabaniños, camino de Piornedo; camino de Tres Obispos (Bilton, 1992).

Citas del resto de España. León (Bilton, 1992).

COMEnTARios. Esta especie presenta un área de distribución muy restringida. 
Oniscus asellus Linnaeus, 1758

Material estudiado. A Coruña: Culleredo, Villa Rutis,

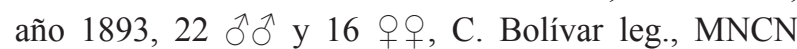

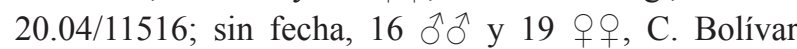
leg. MNCN 20.04/10019. Lugo: Piornedo, Ancares, 25-VII-1952 1 ふ̄, H. Franz leg., col. Schmölzer, MNCN $20.04 / 9666$.

Citas de Galicia. A Coruña: Villa Rutis (Dollfus, 1892). A Capela Caaveiro (Bilton, 1997). Lugo: Baralla, río Ferreira (Bilton, 1997). Puente de Lozera; Piornedo y Sierra de Ancares, Cabana Vella (Schmölzer, 1955a, 1971). Vilar de Donas (Bilton, 1997). Vivero, playa de Covas (Vázquez Felechosa \& Anadón, 2001). Orense: Los Peares (Schmölzer, 1955a, 1971). Pedrosa (Vázquez Felechosa \& Anadón, 2001). Peña Trevinca (Schmölzer, 1955a, 1971). Pontevedra: Bueu (Schmölzer, 1955a, 1971). Carboeiro (Bilton, 1997). Gondomar y Pontevedra (Schmölzer, 1971).

Citas del resto de España. Álava, Asturias, Burgos, Cantabria, Guadalajara, Guipúzcoa, Huesca, La Rioja, León, Lérida, Madrid, Navarra, Vizcaya y Zaragoza (Budde-Lund, 1885; De Buen, 1887; Dollfus, 1892; Racovitza, 1907; Jeannel \& Racovitza, 1918; Arcangeli, 1935; Schmölzer, 1955a, 1955b, 1971; Pablos, 1966; Fidalgo \& Herrera, 1980; Pollo Zorita, 1982, 1986a; Cifuentes, 1984; Vivar et al., 1984; Cruz, 1991; Bilton, 1997; Vázquez Felechosa \& Anadón, 2001; Galán, 2003, 2006; Vázquez Felechosa et al., 2004; Galán \& Nieto, 2011).

Comentarios. Es una especie muy abundante en el norte de España, con algunas poblaciones aisladas en la zona centro.

Oniscus galicianus Bilton, 1997

Citas de Galicia. A Coruña: A Capela Caaveiro (Bilton, 1997). Lugo: Meira; Montes de Valcaloura; Palas de Rei; río Ferreira; río Landro; río Pambe; río Xudan y Vilar de Donas (Bilton, 1997). Pontevedra: As Eiras (Gregory et al., 2012).

COMENTARIOS. Especie endémica de Galicia.

Oniscus lusitanus Verhoeff, 1908

Material estudiado. A Coruña: Finisterre, 10-VIII-1984, $2 \hat{\jmath}$, JC leg. y col.

Citas de Galicia. A Coruña: Outes; río Tambre; San Saturnino y Santiago (Schmölzer, 1971). Orense: Avión y Beade (Gregory et al., 2012). Pontevedra: A Carballeiro (Schmölzer, 1955a). Amorin; As Eiras y Bayona (Gregory et al., 2012). Belus (Schmölzer, 1971). Camposancos (Gregory et al., 2012). Cerdedo (Schmölzer, 1955a, 1971). Goján y Gondomar (Gregory et al., 2012). Marín (Schmölzer, 1955a, 1971). Oia (Gregory et al., 2012). Pontevedra (Schmölzer, 1955a, 1971). Puerto de Moncelos (Gregory et al., 2012). Vigo, Isla Cíes del Norte (Schmölzer, 1971).
Citas del resto de España. Asturias, Cantabria y Vizcaya (Schmölzer, 1971).

COMEnTARios. Es una especie del norte peninsular.

Familia PHILOSCIIDAE Kinahan, 1857

Género Chaetophiloscia Verhoeff, 1908

Chaetophiloscia elongata (Dollfus, 1884)

Citas de Galicia. A Coruña: Villa Rutis (Dollfus, 1892). Orense: Lobios, Puerto de la Home (Schmölzer, 1971).

Citas del resto de España. Alicante, Asturias, Barcelona, Cantabria, Castellón, Cuenca, Gerona, Guadalajara, Guipúzcoa, Islas Baleares, La Rioja, Madrid, Murcia, Navarra, Tarragona, Valencia y Zaragoza (Dollfus, 1892, 1893, 1896, 1897; Arcangeli, 1924, 1925; Schmölzer, 1955a, 1955b, 1971; Vandel, 1961, 1962; Cifuentes, 1984; Vivar et al., 1984; Pollo Zorita, 1986a; Bellés et al., 1989; Cruz, 1989, 1991; García \& Cruz, 1993; Vadell et al., 2006; García, 2009).

Comentarios. Es una especie abundante sobre todo en el área mediterránea española, pero no se ha citado al sur de la línea Lobios (Orense), Madrid y Jávea (Alicante), como también indica Vandel (1962).

Género Ctenoscia Verhoeff, 1928

Ctenoscia minima (Dollfus, 1892)

Material estudiado. Pontevedra: O Grove, 19-VIII-

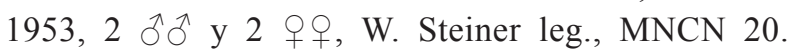
04/9658.

Citas de Galicia (como Ctenoscia dorsalis Verhoeff, 1928). Pontevedra: Camposancos y Oia (Gregory et al., 2012). Pontevedra (Schmölzer, 1955a, 1971).

Citas del resto de España. Cádiz (Schmölzer, 1971), Granada (Dollfus, 1892, 1897; Vandel, 1953), Islas Baleares (Vandel, 1961; Bellés et al., 1989; Cruz, 1989; García \& Cruz, 1996) y Sevilla (Schmölzer, 1971).

Comentarios. Ctenoscia minima presenta una estructura muy característica de los pleópodos del macho (Figs. 1-5) y de la antena del macho (Fig. 6), con el quinto artejo con aspecto ovalado. Taiti \& Rossano (2015) consideran que Ctenoscia dorsalis Verhoeff, 1928 y C. minima, son especies sinónimas, dado que sus diferencias están relacionadas con la talla del ejemplar.

Esta especie de origen atlántico (Vandel, 1946), ha sido escasamente citada de España, aunque es muy común en Portugal, de donde se ha citado de ocho distritos: Braga, Coimbra, Faro, Leiria, Lisboa, Porto, Setúbal y Viana do Castelo (Jackson, 1926; Vandel, 1946; Reboleira et al., 2015). También se encuentra en las islas Canarias, y desde las Baleares hasta Malta, así como en el norte de África (Schmalfuss, 2003; Taiti \& Rossano, 2015). 

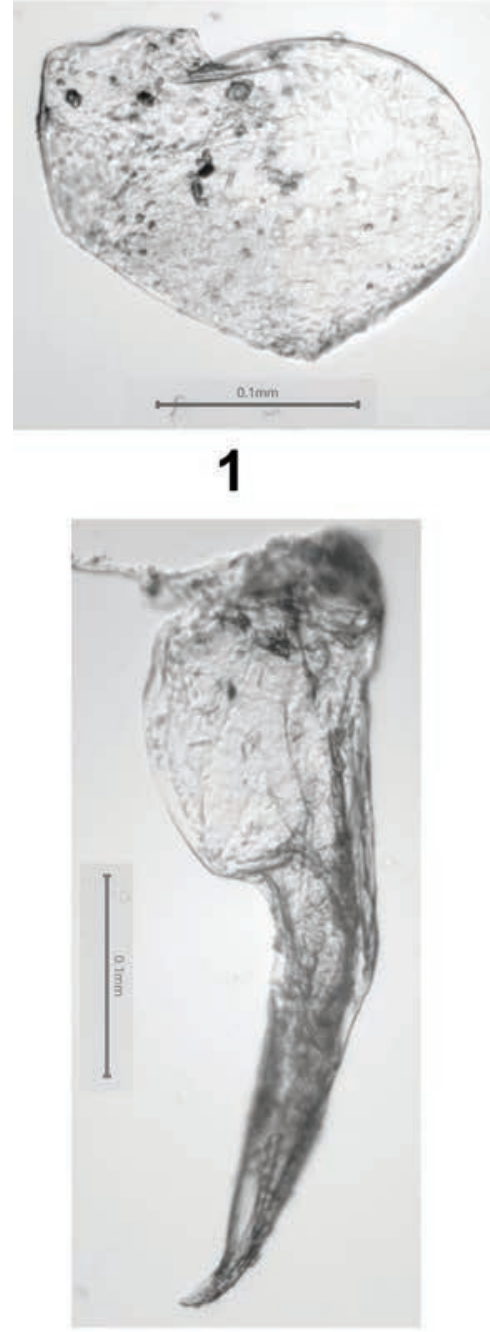

2
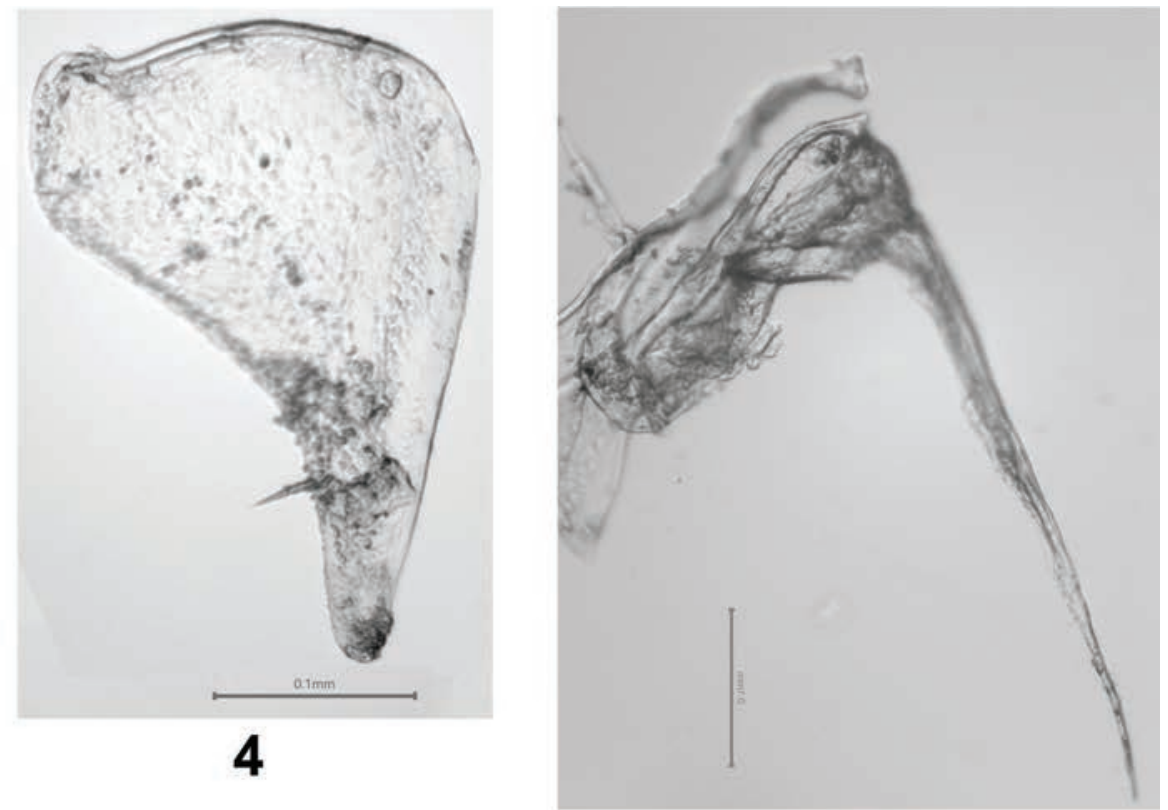

5

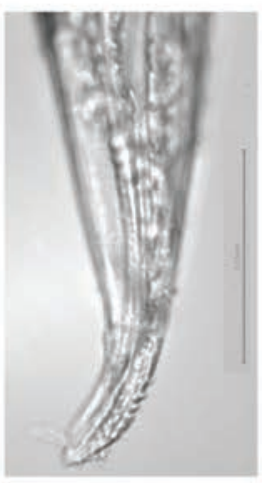

3

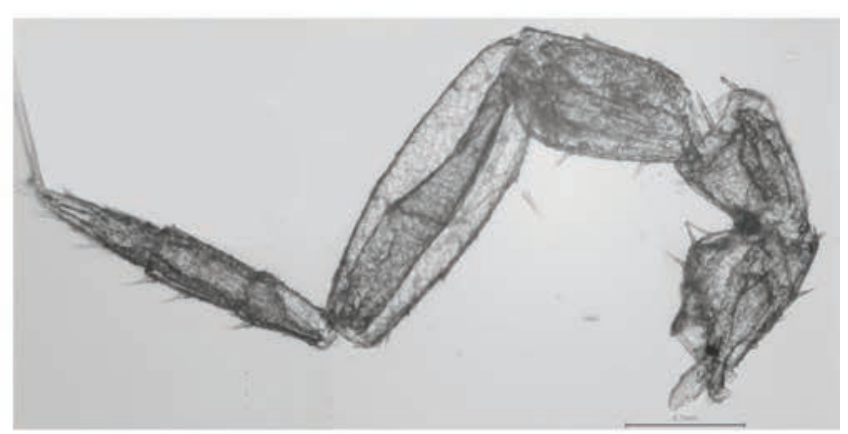

6

Figs. 1-6.- Ctenoscia minima, đ̃: 1: Exopodito I. 2 y 3: Endopodito I. 4: Exopodito II. 5: Endopodito II. 6: Antena.

Figs. 1-6.- Ctenoscia minima, ô: 1: Exopod I. 2 and 3: Endopod I. 4: Exopod II. 5: Endopod II. 6: Antennae.

Género Philoscia Latreille, 1804

Philoscia affinis Verhoeff, 1908

Material estudiado. Lugo: Piornedo (Ancares), 24-VII1952, 1 ふ̋, H. Franz leg., col. Schmölzer, MNCN 20.04/9682.

Citas de Galicia. Lugo: Piornedo (Schmölzer, 1955a). Si bien Schmölzer (1955a) la cita de Piornedo, posteriormente (Schmölzer, 1971), adjudica esta cita a Philoscia muscorum (Scopoli, 1763).

Citas del resto de España. Álava, Asturias, Barcelona, Burgos, Cantabria, Gerona, Guipúzcoa, Islas Baleares, La Rioja, Navarra, Tarragona y Vizcaya (Schmölzer, 1955a, 1971; Vandel, 1962; Fidalgo \& Herrera, 1980; Cifuentes, 1984; Vivar et al., 1984; Cruz, 1991; García \& Cruz, 1996; Vázquez Felechosa et al., 2004). Por otra parte, Vandel (1962) pone en duda las citas de Arcangeli $(1924,1925)$ de Barcelona y Gerona de $P h$. muscorum, y las atribuye a $P h$. affinis.
Comentarios. Vandel (1962), también pone en duda las citas de Ph. muscorum de Portugal: Estoril (Jackson, 1926), Sintra (Preudhomme De Borre, 1886) y Coimbra (Carvalho, 1944 según Vandel, 1962), indicando que no representan poblaciones autóctonas.

El ejemplar estudiado de la colección Schmölzer del MNCN, tiene los mismos datos de captura que los publicados por Schmölzer (1955a, 1971), aunque es un macho y los que indica este autor en sus trabajos son 2 hembras. Por ello consideramos que $P h$. muscorum no se encuentra en Galicia. Philoscia affinis es una especie que se encuentra en la zona norte peninsular.

Familia HALOPHILOSCIIDAE Verhoeff, 1908

Género Halophiloscia Verhoeff, 1908

Halophiloscia couchii (Kinahan, 1858)

Citas de Galicia. Pontevedra: Oia (Gregory et al., 2012). 
Citas del Resto de España. Alicante (Cruz, 1992), Barcelona (Cruz, 1991, 1992), Cádiz (Schmölzer, 1955b), Cantabria (Schmölzer, 1971), Castellón (Cruz, 1992), Guipúzcoa (Dollfus, 1892, 1896, 1897), Islas Baleares (Budde-Lund, 1885; Cruz, 1992; García \& Cruz, 1996; García, 2008, 2009) y Tarragona (Cruz, 1992).

Comentarios. Es una especie halófila, que ocupa el área supramareal.

Familia PLATYARTHRIDAE Verhoeff, 1949

Género Platyarthrus Brandt, 1833

Platyarthrus hoffmannseggii Brandt, 1833

Citas de Galicia. A Coruña: Villa Rutis (Dollfus, 1892).

Pontevedra: Bayona (Gregory et al., 2012).

CitAs del RESTO DE EsPAÑA. Álava (Vivar et al., 1984), Cuenca (Pollo Zorita, 1986a), Guadalajara (Pollo Zorita, 1986a), Guipúzcoa (Vandel, 1962), Lérida (Arcangeli, 1925), Navarra (Vandel, 1962; Fidalgo \& Herrera, 1980; Cifuentes, 1984).

Comentarios. Esta especie mirmecófila de origen mediterráneo (Vandel, 1962), ha sido muy escasamente citada de la península.

Familia PORCELLIONIDAE Brandt \& Ratzeburg, 1831 Género Porcellionides Miers, 1877

Porcellionides buddelundi (Verhoeff, 1901)

Citas de Galicia. A Coruña: Muros y Noya, Sierra de Barbanza (Schmölzer, 1971). Orense: Lobios, Puerto de la Home (Schmölzer, 1971). Pontevedra: Bayona ctra. La Guardia (Schmölzer, 1971). Marín (Schmölzer, 1955a, 1971).

Citas del Resto de España. Granada (Schmölzer, 1955a).

Comentarios. La citas de P. buddelundi de Loja y Silla del Moro (Granada) de Schmölzer (1955a) probablemente deben de ser revisadas, ya que estas localidades quedan muy lejos del área de distribución de esta especie. La cita más meridional corresponde a Coimbra (Verhoeff, 1901; Arcangeli, 1936).

Porcellionides cingendus (Kinahan, 1857)

Material estudiado. A Coruña: Finisterre, 10-VIII-1984, $2 \hat{\jmath} \widehat{o}$, JC leg. y col.

Citas de Galicia. A Coruña: Villa Rutis (Dollfus, 1893). Orense: Avión; Beade y Beiro (Gregory et al., 2012). Los Peares y Sobradelo (Schmölzer, 1955a, 1971). Pontevedra: Amorin; As Eiras; Bayona; Camposancos; Goján; Oia y Puerto de Moncelos (Gregory et al., 2012).

Citas del Resto de España. Asturias (Vandel, 1962), Cantabria (Dollfus, 1892; Vivar et al., 1984) y Vizcaya (Vivar et al., 1984).

Comentarios. Es una especie del norte de España y Portugal, en este país ha sido citada de los distritos de Braga (Vandel, 1946), Coimbra (Arcangeli, 1936; Vandel, 1946), Leiria y
Lisboa (Vandel, 1946), Porto (Arcangeli, 1936; Vandel, 1946) y Viana do Castelo (Gregory et al., 2012).

Porcellionides pruinosus (Brandt, 1833)

Citas de Galicia. A Coruña: Villa Rutis (Dollfus, 1892).

Pontevedra: Oia (Gregory et al., 2012).

Citas del Resto de España. Almería, Barcelona, Cádiz, Castellón, Ciudad Real, Cuenca, Gerona, Granada, Guadalajara, Guipúzcoa, Huelva, Islas Baleares, Islas Canarias, Lérida, Madrid, Málaga, Navarra, Sevilla, Toledo y Zaragoza (Budde-Lund, 1885; De Buen, 1887; Dollfus, 1892, 1897; Arcangeli, 1924, 1925; Vandel, 1953, 1961; Schmölzer, 1955a, 1971; Fidalgo \& Herrera, 1980; Cifuentes, 1984; Vivar et al., 1984; Pollo Zorita, 1986a; Bellés et al., 1989; Cruz, 1989, 1991; García \& Cruz, 1993; Vadell, 2003; Vadell \& Zaragoza, 2005; García, 2008, 2009).

Comentarios. Es una especie de amplia distribución en la península ibérica y también mundial.

Porcellionides rufocinctus (Dollfus, 1892)

Citas de Galicia. A Coruña: Villa Rutis y San Roque (Dollfus, 1893).

Comentarios. Es una especie endémica de Galicia, que hasta el presente no ha vuelto a ser citada.

Porcellionides sexfasciatus (Budde-Lund, 1885)

Material estudiado. A Coruña: Villa Rutis, 1893, 1 ō, C. Bolívar leg., MNCN 20.04/11518. Pontevedra: Portonovo, 30-VII-1985, 4 ふึ઼, 2 우 y 9 inmaduros, JC leg. y col.

Citas de Galicia. Lugo: Puente de Lozera (Schmölzer, 1955a). Pontevedra: Pontevedra (Schmölzer, 1955a).

Citas del resto de España. Alicante, Almería, Barcelona, Cádiz, Castellón, Ciudad Real, Córdoba, Gerona, Granada, Guipúzcoa, Huelva, Islas Baleares, Jaén, Madrid, Málaga, Navarra, Sevilla y Vizcaya (Budde-Lund, 1885; Dollfus, 1892; Schmölzer, 1955a, 1955b, 1971; Español, 1958; Vandel, 1961, 1962; Pablos, 1964; Cifuentes, 1984; Vivar et al., 1984; Pollo Zorita, 1986a, 1986b; Bellés et al., 1989; Cruz, 1989, 1991; García \& Cruz, 1993, 1996; Vadell, 2003; Vadell \& Zaragoza, 2005; Vadell et al., 2006; García, 2008).

Comentarios. Es una especie ampliamente repartida por la península ibérica.

Porcellionides sexfasciatus lusitanus (Vandel, 1945)

Citas de Galicia. A Coruña: Corme, bahía, y Noya, Sierra de Barbanza (Schmölzer, 1971). Pontevedra: Camposancos (Gregory et al., 2012). Bayona, Monte Ferro; Bueu, Isla de Ons; Gondomar; Lourido y Vigo, Isla Cíes del Norte (Schmölzer, 1971).

Citas del resto de España. Cádiz (Cruz, 1991), Madrid (Schmölzer, 1955a) y Málaga (Cruz, 1991).

Comentarios. Esta subespecie ha sido citada de algunas localidades de la mitad oriental peninsular. 
Porcellionides sexfasciatus molleri (Verhoeff, 1901)

Material estudiado. Pontevedra: La Guardia, 3-VIII-

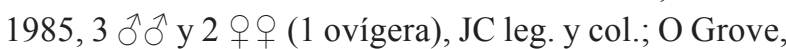
6-VIII-1953, 17 ふ઼ y 17 우 (7 ovígeras), W. Steiner leg., col. Schmölzer, MNCN 20.04/9661; 18-VIII1953, 1 गे, W. Steiner leg., col. Schmölzer, MNCN $20.04 / 9631$.

Citas de Galicia. A Coruña: Corrubedo ctra. Olveira; Mañón, Punta de la Estaca; Noya, Sierra de Barbanza y Santiago, río Tambre (Schmölzer, 1971). Lugo: Puente de Lozera (Schmölzer, 1955a). Pontevedra: Bueu, Castiñeira y Pontevedra (Schmölzer, 1955a). Bayona, Cabo Silleiro y Sierra de la Grova; Bueu, Isla de Ons; Cangas de Morrazo; Gondomar; La Lamosa, Sierra de la Frontefria; Marín; Pontevedra y Vigo, ría (Schmölzer, 1971).

Comentarios. Esta subespecie solamente se encuentra en la zona más occidental de la península ibérica, se ha citado de Galicia y de los distritos portugueses de Coimbra, Faro, Leiria, Lisboa, Setúbal y Viseu (Verhoeff, 1901; Arcangeli, 1936; Vandel, 1946; Schmölzer, 1971).

Género Porcellio Latreille, 1804

Porcellio debueni Dollfus, 1892

Material estudiado. A Coruña: A Coruña, El Burgo, 10-VIII-1892, 4 ふَ̋̄ y 11 우, MNCN 20.04/7417; Pasaje, 14-IX-1892, 1 ઈ y 2 우, MNCN 20.04/9786. Pontevedra, O Grove, 19-VIII-1953, 1 ठै y 5 우 (3 ovígeras), W. Steiner leg., col. Schmölzer, MNCN 20.04/9629; 19-VIII-1953, 1 त̂̀ y 1 o , W. Steiner leg., col. Schmölzer, MNCN 20.04/9641; 27-VIII-1953, 1 †े y 2 우, W. Steiner leg., col. Schmölzer, MNCN 20.04/9678; 31-VIII-1953, 1 ô y 4 ㅇ, W. Steiner leg., MNCN 20.04/9664.

Citas de Galicia. A Coruña: El Burgo (Dollfus, 1893). Villa Rutis (Dollfus, 1892). Orense: Avión, río Valdeiras y Beade (Gregory et al., 2012). Lobios, Puerto de la Home - Sierra de Gêres (Schmölzer, 1971). Pontevedra: Amorin y As Eiras (Gregory et al., 2012). Bayona (Cabo Silleiro, Sierra de la Grova, Isla Estela) (Schmölzer, 1971). Bayona; Camposancos y Goján (Gregory et al., 2012). Gondomar y La Caniza, Sierra de Fontefria (Schmölzer, 1971). Oia (Gregory et al., 2012). O Grove (Cifuentes, 2018). Vigo, Isla Cíes del Norte (Schmölzer, 1971).

Citas del Resto de España. Asturias (Schmölzer, 1971; Vázquez Felechosa et al., 2004) y León (Pollo Zorita, 1982; Cifuentes, 2018).

Comentarios. Es una especie del centro y norte de Portugal, Galicia y León. La cita de Málaga de esta especie (Cifuentes, 2018), se debe a un error en el etiquetado de los ejemplares MNCN 20.04/7417. El etiquetado correcto es A Coruña, El Burgo, 10-VIII-1892, en vez de Málaga, El Burgo, 10-VIII-1992.
Porcellio dilatatus Brandt, 1833

Material estudiado. A Coruña: A Coruña, 5-IV-1907, 1 q, C. Bolívar leg., MNCN 20.04/9880. Villa Rutis,

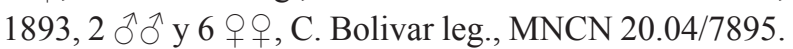
Pontevedra, O Grove, Playa A Lanzada., 2-VII-1952, 1 ô, H. Franz leg., MNCN 20.04/9628.

Citas de Galicia. A Coruña: Corme, bahía (Schmölzer, 1971). El Burgo (Dollfus, 1893). Villa Rutis (Dollfus, 1892). Pontevedra: Oia (Gregory et al., 2012). Pontevedra (Schmölzer, 1955a).

Citas del resto de España. Alicante, Almería, Asturias, Barcelona, Cádiz, Cuenca, Granada, Guadalajara, Huesca, Islas Baleares, León, Lérida, Madrid, Málaga, Navarra, Sevilla y Tarragona (Dollfus, 1892; Arcangeli, 1925, 1935; Jeannel \& Racovitza, 1929; Vandel, 1951, 1953, 1961, 1962; Coiffait, 1959; Schmölzer, 1971; Pollo Zorita, 1982, 1986a; Cifuentes, 1984, 2018; Bellés et al., 1989; Cruz, 1989, 1991; García \& Cruz, 1996).

Comentarios. Porcellio dilatatus es una especie de amplia dispersión que ha sido introducida en muchas partes del mundo.

\section{Porcellio dispar Verhoeff, 1901}

Citas de Galicia. A Coruña: Santiago, bosque de Cernadas (Schmölzer, 1955a, 1971). Pontevedra: A Carballeiro (Schmölzer, 1971). Oia (Gregory et al., 2012). Pontevedra (Schmölzer, 1955a).

Citas del Resto de España. Asturias (Schmölzer, 1955a, 1971), Guadalajara (Cifuentes, 2018).

Comentarios. Esta especie presenta una distribución muy poco conocida.

\section{Porcellio gallicus Dollfus, 1904}

Citas de Galicia. Lugo: Santalla de Lozera (Schmölzer, 1955a).

CitAs del Resto de España. Álava (Cifuentes, 2018), Guipúzcoa (Dollfus, 1892; Schmölzer, 1955a, 1971; Cifuentes, 2018), Navarra (Vandel, 1962; Schmölzer, 1971; Fidalgo \& Herrera, 1980; Cifuentes, 1984, 2018; Vivar et al., 1984) y Vizcaya (Vivar et al., 1984).

Comentarios. Esta especie se encuentra en gran parte de Francia, en alguna localidad de Suiza y en la península ibérica en unas pocas localidades del país vasco y Navarra. La cita de Lugo sería la más occidental en la península.

\section{Porcellio herminiensis Vandel, 1946}

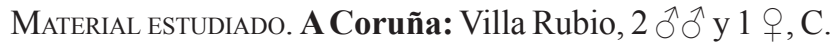
Bolívar leg., MNCN 20.04/9861. Villa Rutis, 1893, 1 đૈ y 8 우, C. Bolívar leg., MNCN 20.04/11517. Pontevedra, O Grove, 19-VIII-1953, 6 inmaduros, W. Steiner leg., MNCN 20.04/11522; 27-VIII-1953, 3 qq, W. Steiner 
leg., MNCN 20.04/9714. Pontevedra, 3-VII-1952, 1 ふૈ, H. Franz leg., col. Schmölzer, MNCN 20.04/9684.

Citas de Galicia. Lugo: Puente Lozera (Schmölzer, 1955a, 1971). Orense: Avión, río Valdeiras; Beade y Beiro (Gregory et al., 2012). Pontevedra: Pontevedra (Schmölzer, 1955a, 1971; Cifuentes, 2018). Puerto de Moncelos (Gregory et al., 2012).

Comentarios. El área de distribución de $P$. herminiensis se limita a Galicia y a tres distritos portugueses, Guarda, Porto y Viana do Castelo (Vandel, 1946; Gregory et al., 2012).

Porcellio incanus Budde-Lund, 1885

Citas de Galicia. Pontevedra: Bayona, Isla Estela (Schmölzer, 1971).

Citas del resto de España. Álava, Alicante, Barcelona, Cádiz, Castellón, Córdoba, Cuenca, Gerona, Guadalajara, Huesca, Islas Baleares, Jaén, La Rioja, León, Lérida, Madrid, Málaga, Murcia, Navarra, Sevilla, Tarragona, Teruel, Valencia y Vizcaya (Racovitza, 1907; Arcangeli, 1924, 1935; Vandel, 1951, 1952; Schmölzer, 1955b, 1971; Fidalgo \& Herrera, 1980; Cifuentes, 1984, 2018; Vivar et al., 1984; Pollo Zorita, 1986a; Cruz, 1991; García \& Cruz, 1996; García, 2008, 2013; González Silvestre, 2015; Cifuentes \& Tinaut, 2018).

Comentarios. Es una especie muy común en la península ibérica.

Porcellio laevis Latreille, 1804

Citas de Galicia. A Coruña: Corme, bahía (Schmölzer, 1971).

Citas del resto de España. Alicante, Almería, Barcelona, Cádiz, Ciudad Real, Córdoba, Cuenca, Gerona, Granada, Guadalajara, Guipúzcoa, Huelva, Huesca, Islas Baleares, Jaén, La Rioja, Lérida, Madrid, Málaga, Murcia, Navarra, Sevilla, Tarragona, Vizcaya y Zaragoza (Budde-Lund, 1885; De Buen, 1887; Dollfus, 1892, 1893, 1897; Racovitza, 1907; Arcangeli, 1925; Jackson, 1926; Vandel, 1953, 1961; Schmölzer, 1955a, 1971; Fidalgo \& Herrera, 1980; Cifuentes, 1984, 2018; Vivar et al., 1984; Pollo Zorita, 1986a; Bellés et al., 1989; Cruz, 1989, 1991; García \& Cruz, 1996; Vadell et al., 2006; García, 2009; Cifuentes \& Tinaut, 2018).

Comentarios. Es una especie cosmopolita.

Porcellio monticola Lereboullet, 1853

Citas de Galicia. Lugo: Puente de Lozera (Schmölzer, 1955a). Citas del Resto de España. Álava, Asturias, Barcelona, Burgos, Castellón, Cuenca, Gerona, Granada, Guadalajara, Huesca, La Rioja, León, Lérida, Madrid, Navarra, Soria, Tarragona, Teruel y Zaragoza (Budde-Lund, 1885; Dollfus, 1892, 1893; Schmölzer, 1955a, 1971; Vandel, 1958, 1962; Fidalgo \& Herrera, 1980; Pollo Zorita, 1982, 1986a; Cifuentes, 1984, 2018; Vivar et al., 1984; Cruz, 1991).
Comentarios. Es una especie ampliamente extendida por la mitad norte peninsular.

\section{Porcellio olivieri (Audouin, 1826)}

Citas de Galicia. Orense: Sobradelo (Schmölzer, 1955a).

Citas del Resto de España. Asturias, Granada y Zaragoza (Schmölzer, 1955a).

Comentarios. Porcellio olivieri es una especie del norte de África, por lo que las citas de Schmölzer (1955a) deben de ser un error de determinación.

\section{Porcellio scaber Latreille, 1804}

Material estudiado. A Coruña: Santiago de Compostela, 12-XI-1969, 3 우, MNCN 20.04/8109. Villa Rubio, 1 옹, C. Bolívar leg., MNCN 20.04/11512. Villa Rutis, 1893, 4 츃 y 6 우, C. Bolivar leg., MNCN 20.04/11519.

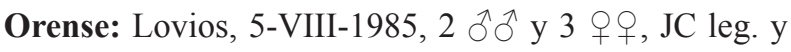
col. Pontevedra: Cabo de Hornos, 31-VII-1985, 1 సै y 1 q, JC leg. y col. O Grove, 8-VIII-1953, 1 \%, W. Steiner leg., col. Schmölzer, MNCN 20.04/9668; 27-VIII-1953, 8 inmaduros, W. Steiner leg., MNCN 20.04/9678. Portonovo, 31-VII-1985, 1 ๙ y 1 ㅇ, JC leg. y col.

Citas de Galicia. A Coruña: Noya (Schmölzer, 1971). San Saturnino ctra. Vivero (Schmölzer, 1971). Villa Rutis (Dollfus, 1892). Lugo: Mustallar; Peña Rubia; Piornedo, bosque del río da Freito y Sierra de Ancares, Cabana Vella (Schmölzer, 1971). Orense: Sierra de San Mamede, Montes del Invernadeiro (Schmölzer, 1971). Pontevedra: Amorin y As Eiras (Gregory et al., 2012). Bayona, Isla Estela (Schmölzer, 1971). Bayona (Gregory et al., 2012). Camposancos (Gregory et al., 2012). Fuenfría (Dollfus, 1892, 1897). Goján, río Miño y Oia (Gregory et al., 2012). Vigo (Dollfus, 1892, 1897). Vigo, Isla Cíes del Norte e Isla Cíes del Sur (Schmölzer, 1971). Citas del Resto DE EspañA. Álava, Asturias, Ávila, Burgos, Cáceres, Cantabria, Cuenca, Gerona, Granada, Guadalajara, Guipúzcoa, Huesca, La Rioja, León, Lérida, Madrid, Murcia, Navarra, Soria, Tarragona, Teruel, Vizcaya y Zaragoza (De Buen, 1887; Dollfus, 1892, 1893, 1897; Arcangeli, 1925; Vandel, 1953; Schmölzer, 1971; Fidalgo \& Herrera, 1980; Pollo Zorita, 1982, 1986a; Cifuentes, 1984, 2018; Vivar et al., 1984; Cruz, 1991; Galán, 2003; Vázquez Felechosa et al., 2004; Galán \& Nieto, 2011).

Comentarios. Porcellio scaber es una especie cosmopolita.

\section{Porcellio scaber "forma" lusitanus Verhoeff, 1907}

Material estudiado. Pontevedra: La Guardia, 3-VIII-1985, 1 đ̊ y 3 우 (1 ovígera), JC leg. y col.; O Grove, 8-VIII-

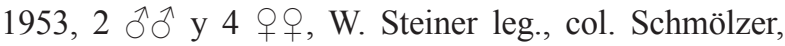
MNCN 20.04/9670; 8-VIII-1953, 1 oे, W. Steiner leg., MNCN 20.04/9679; 8-VIII-1953, 5 ふึતึ y 9 우, W. Steiner leg., col. Schmölzer, MNCN 20.04/9642; 9-VIII1953, 1 q, W. Steiner leg., col. Schmölzer, MNCN 
20.04/9646; 18-VIII-1953, 1 đ̄ y 1 ㅇ, W. Steiner leg., MNCN 20.04/9680; 18-VIII-1953, 5 inmaduros, W. Steiner leg., col. Schmölzer, MNCN 20.04/9677; 20-VIII1953, 13 inmaduros, W. Steiner leg., col. Schmölzer, MNCN 20.04/9676; 27-VIII-1953, 1 J, W. Steiner leg., col. Schmölzer, MNCN 20.04/9678.

Citas de Galicia. A Coruña: Malpica, Punta de la Boca; Mañón, Punta de la Estaca, Faro; Noya, Sierra de Barbanza y Ortigueira, El Baraquera (Schmölzer, 1971). Santiago, bosque de Cernadas (Schmölzer, 1955a, 1971) y orilla del río Tambre (Schmölzer, 1971). Lugo: Puente de Lozera (Schmölzer, 1971). Piornedo, bosque del río da Freito (Schmölzer, 1955a). Orense: Lobios, Sierra de Gêres (Schmölzer, 1971). Los Peares y Peña Trevinca (Schmölzer, 1955a, 1971). Sierra de San Mamede, Montes del Invernadeiro (Schmölzer, 1971). Pontevedra: A Carballeiro, Bayona, Cabo Silleiro, Isla Estela, Monte Ferro y Sierra da A Groba (Schmölzer, 1971). Bayona, Virgen de la Boca (Schmölzer, 1955a, 1971). Bueu, Isla de Ons; Figuerido; Gondomar y La Caniza-A Lamosa, Sierra de Fontefria (Schmölzer, 1971). La Guardia, delta Miño (Schmölzer, 1955a, 1971). La Guardia, punta Santa Tecla (Cifuentes, 2018). Marín (Schmölzer, 1971). Mondariz (Schmölzer, 1955a, 1971). Nigrán, Monteferro (Schmölzer, 1971). O Grove, playa La Lanzada (Schmölzer, 1955a, 1971). O Grove (Cifuentes, 2018). Pontevedra (Schmölzer, 1955a, 1971). Testeiro (Schmölzer, 1955a, 1971). Vigo, Isla Cíes del Norte e Isla Cíes del Sur (Schmölzer, 1971).

Citas del resto de España. Asturias, Cádiz, Cuenca, Guadalajara, Guipúzcoa, Huesca-Zaragoza, Madrid y Teruel (Schmölzer, 1955a; Pollo Zorita, 1986a).

Comentarios. Pollo Zorita $(1982,1983)$ ya indicó que “lusitanus" es solamente una forma en el sentido de Vandel, 1962, que se presenta solamente en los ejemplares de gran talla, por efecto del crecimiento alométrico, por lo que debe de indicarse como Porcellio scaber "forma" lusitanus.

Familia ARMADILLIDIIDAE Brandt, 1833

Género Armadillidium Brandt, 1833

Armadillidium album Dollfus, 1887

Citas de Galicia. A Coruña: Ortigueira, playa de Morouzos (García, 2005).

Citas del Resto de España. Almería (Cruz, 1993), Islas Baleares y Valencia (García et al., 2003).

Comentarios. La distribución de esta especie halófila es todavía muy poco conocida.

Armadillidium nasatum Budde-Lund, 1885

Citas de Galicia. Galicia: sin localidad (Vandel, 1962).

Orense: Seoane (Vázquez Felechosa \& Anadón, 2001).

Citas del resto de España. Asturias (Schmölzer, 1955a, 1971; Vandel, 1962; Vázquez Felechosa \& Anadón, 2001), Cantabria (Vivar et al., 1984; Vázquez
Felechosa \& Anadón, 2001), Gerona (Cruz, 1993), Guipúzcoa (Dollfus, 1892; Schmölzer, 1955a, 1955b, 1971), Navarra (Dollfus, 1892; Fidalgo \& Herrera, 1980; Cifuentes, 1984; Vivar et al., 1984; Cruz, 1993) у Vizcaya (Vivar et al., 1984).

COMENTARios. En la penísula ibérica esta especie se encuentra localizada en una estrecha franja entre Galicia y pirineos, siendo las citas más orientales las de la provincia de Gerona.

\section{Armadillidium pictum Brandt, 1833}

Material estudiado. Pontevedra: Cerdedo, 31-VII-1952, 1 †, H. Franz leg., MNCN 20.04/9634.

Citas de Galicia. Lugo: Puente de Lozera y Piornedo, bosque del río da Freito (Schmölzer, 1955a, 1971).

Citas del RESto DE España. Asturias (Cruz, 1991, 1993; Vázquez Felechosa \& Anadón, 2001), Huesca (Schmölzer, 1971), Lérida (Cruz, 1993) y Navarra (Schmölzer, 1971; Fidalgo \& Herrera, 1980; Vivar et al., 1984; Cruz, 1993).

Comentarios. Su amplia distribución europea contrasta con la mucho más limitada en la península ibérica, de donde ha sido citada para algunas provincias del norte. Schmölzer (1955a) describió la especie A. galiciense Schmölzer, 1955, con ejemplares gallegos, mencionando a Cerdedo como localidad tipo (Schmölzer, 1955a, 1971). De esta localidad se ha podido estudiar 1 ejemplar (MNCN 20.04/9634) con los mismos datos de captura que los de este autor, y pertenece a la especie A. pictum, como así lo había indicado Vandel (1962).

Armadillidium sordidum Dollfus, 1887

Citas de Galicia. Pontevedra: Pontevedra (Schmölzer, 1955a).

Citas del Resto de España. Valencia (Cruz, 1993).

Comentarios. La cita de esta especie de Pontevedra debe de ser un error de determinación, dado que es una especie del noroeste de Italia, Cerdeña, Córcega y algunas localidades del sureste de Francia (Vandel, 1962; Schmalfuss, 2003). En España solamente es correcta la cita de Cruz (1993).

\section{Armadillidium vulgare (Latreille, 1804)}

Material estudiado. Lugo: Lugo, VIII-1959, 1 q, MNCN 20.04/8162. Pontevedra: O Grove, 8-VIII-1953, 1 q, W. Steiner leg., MNCN 20.04/9927; 19-VIII-1953, 1 ㅇ, W. Steiner leg., MNCN 20.04/9933; 27-VIII-1953, 1 q y 26 inmaduros, W. Steiner leg., MNCN 20.04/9946. Pontevedra, 1-VII-1952, 1 q, H. Franz leg., MNCN 20.04/9948

Citas de Galicia. A Coruña: Noya, Sierra de Barbanza (Schmölzer, 1971). Santiago, orilla del Rio Tambre (Schmölzer, 1971). Lugo: Vivero, playa de Covas (Vázquez Felechosa \& Anadón, 2001). Orense: Avión, 
valle río Valdeiras (Gregory et al., 2012). Baños de Molgas, Guamil y Seoane (Vázquez Felechosa \& Anadón, 2001). Pontevedra: As Eiras (Gregory et al., 2012). Bayona (Gregory et al., 2012). Bueu, Isla de Ons (Schmölzer, 1971). Camposancos (Gregory et al., 2012). Cangas de Morrazo, Barra (Schmölzer, 1971). Cerdedo (Schmölzer, 1955a, 1971). Goján, río Miño y Oia (Gregory et al., 2012). Pontevedra (Schmölzer, 1955a, 1971). Vigo (Schmölzer, 1971).

Citas del resto de España. Álava, Alicante, Almería, Asturias, Ávila, Barcelona, Burgos, Cádiz, Cantabria, Ciudad Real, Cuenca, Gerona, Granada, Guadalajara, Guipúzcoa, Huelva, Islas Baleares, Jaén, La Rioja, León, Lérida, Madrid, Málaga, Murcia, Navarra, Sevilla, Soria, Tarragona, Teruel, Toledo, Valladolid, Vizcaya, Zamora, y Zaragoza (Koch, 1856; De Buen, 1887; Arcangeli, 1924, 1925; Jackson, 1926; Vandel, 1953, 1961; Schmölzer, 1955a, 1971; Fidalgo \& Herrera, 1980; Pollo Zorita, 1982, 1986a, 1986b; Cifuentes, 1984; Vivar et al., 1984; Cruz, 1991, 1993; García \& Cruz, 1993; Vázquez Felechosa \& Anadón, 2001; García, 2008, 2009; Cifuentes \& Tinaut, 2018).

Comentarios. Armadillidium vulgare es una especie cosmopolita.

Género Eluma Budde-Lund, 1885

Eluma caelata (Miers, 1877)

Citas de Galicia. A Coruña: Finisterre, Cabo de Finisterre (Schmölzer, 1971). Mañón, Punta de la Estaca (Schmölzer, 1971). Noya, Serra de Barbanza y Serra de Outes (Schmölzer, 1971). Santiago, bosque de Cernadas (Schmölzer, 1955a, 1971); orilla del Rio Tambre (Schmölzer, 1971). Lugo: Puente de Lozera (Schmölzer, 1955a, 1971). Sierra de Ancares, Peña Rubia (Schmölzer, 1971). Orense: Avión, valle río Valdeiras; Beade y Beiro (Gregory et al., 2012). Los Peares, río Miño (Schmölzer, 1955a, 1971). Lobios (Schmölzer, 1971); Puerto de la Home (Schmölzer, 1971). Pontevedra: A Carballeiro (Schmölzer, 1955a). Amorin; As Eiras; Bayona y Camposancos (Gregory et al., 2012). Cerdedo (Schmölzer, 1955a, 1971). Figuerido y Gondomar (Schmölzer, 1971). Goján, río Miño (Gregory et al., 2012). Pontevedra (Schmölzer, 1955a, 1971). Oia y Puerto de Moncelos (Gregory et al., 2012).

Citas del resto de España. Álava, Almería, Asturias, Cádiz, Cantabria, Córdoba, Granada, Jaén, La Rioja, Málaga, Navarra, Sevilla y Vizcaya (Dollfus, 1892; Arcangeli, 1936; Vandel, 1953, 1954, 1962; Schmölzer, 1955a, 1955b, 1971; Cifuentes, 1984; Vivar et al., 1984; Vázquez Felechosa \& Anadón, 2001; Vázquez Felechosa et al., 2004; García, 2013; García \& Pérez Fernández, 2017).

Comentarios. Es una especie de amplia dispersión en la península ibérica, de donde es originaria según Vandel (1962).

\section{Discusión}

Como ya se ha indicado, hasta el presente trabajo se habían citado 43 especies de Galicia, y por diferentes razones siete de ellas deben de ser eliminadas del censo de isópodos gallegos, estas son:

Tabla 1.- Lista sistemática de las especies de isópodos terrestres gallegos por provincias.

Table 1.- Systematic list of Galician terrestrial isopod species by provinces.

\begin{tabular}{|c|c|c|c|c|}
\hline Especie & A Coruña & Lugo & Orense & Pontevedra \\
\hline Tylos europaeus & & & & $x$ \\
\hline Ligia oceanica & $x$ & & & $x$ \\
\hline Haplophthalmus danicus & & & $x$ & $x$ \\
\hline Miktoniscus bisetosus & & & & $x$ \\
\hline Miktoniscus patiencei & & & & $x$ \\
\hline Oritoniscus simplex & $x$ & $x$ & & $x$ \\
\hline Trichoniscoides ancaresi & & $x$ & & \\
\hline Trichoniscoides lusitanus & $x$ & & $x$ & $x$ \\
\hline Trichoniscus provisorius & $x$ & & $x$ & $x$ \\
\hline Trichoniscus pusillus & $x$ & & $x$ & $x$ \\
\hline Oniscus ancarensis & & $x$ & & \\
\hline Oniscus asellus & $x$ & $x$ & $x$ & $x$ \\
\hline Oniscus galicianus & $x$ & $x$ & & $x$ \\
\hline Oniscus lusitanus & $x$ & & $x$ & $x$ \\
\hline Chaetophiloscia elongata & $x$ & & $x$ & \\
\hline Ctenoscia minima & & & & $x$ \\
\hline Philoscia affinis & & $x$ & & \\
\hline Halophiloscia couchii & & & & $x$ \\
\hline Platyarthrus hoffmannseggii & $x$ & & & $x$ \\
\hline Porcellionides buddelundi & $x$ & & $x$ & $x$ \\
\hline Porcellionides cingendus & $x$ & & $x$ & $x$ \\
\hline Porcellionides pruinosus & $x$ & & & $x$ \\
\hline Porcellionides rufocinctus & $x$ & & & \\
\hline Porcellionides sexfasciatus & $x$ & $x$ & & $x$ \\
\hline Porcellio debueni & $x$ & & $x$ & $x$ \\
\hline Porcellio dilatatus & $x$ & & & $x$ \\
\hline Porcellio dispar & $x$ & & & $x$ \\
\hline Porcellio gallicus & & $x$ & & \\
\hline Porcellio herminiensis & $x$ & $x$ & $x$ & $x$ \\
\hline Porcellio incanus & & & & $x$ \\
\hline Porcellio laevis & $x$ & & & \\
\hline Porcellio monticola & & $x$ & & \\
\hline Porcellio scaber & $x$ & $x$ & $x$ & $x$ \\
\hline Armadillidium album & $x$ & & & \\
\hline Armadillidium nasatum & & & $x$ & \\
\hline Armadillidium pictum & & $x$ & & $x$ \\
\hline Armadillidium vulgare & $x$ & $x$ & $x$ & $x$ \\
\hline Eluma caelata & $x$ & $x$ & $x$ & $x$ \\
\hline
\end{tabular}


Oritoniscus flavus, cuyos ejemplares corresponden en realidad a $O$. simplex.

Trichoniscoides modestus, es una especie del sur de Francia, por lo que la cita debe de ser confirmada según Schmalfus (2003).

Philoscia muscorum, ya que en realidad es Philoscia affinis según la revisión realizada.

Ctenoscia dorsalis al tratarse de una sinonimia de Ctenoscia minima.

Porcellio olivieri es una especie del norte de África, por lo que las citas deben de ser un error de determinación.

Armadillidium galiciense es un error de determinación, se trata de $A$. pictum.

Armadillidium sordidum también debe de ser un error de determinación, dado que es una especie del mediterráneo occidental.

La revisión de la colección de isópodos del Museo Nacional de Ciencias Naturales de Madrid (MNCN) y del autor, ha permitido aportar nuevos datos de 19 especies, e incorporar una de ellas a la lista de isópodos terrestres gallegos al citarse por primera vez en este trabajo, Tylos europaeus para Pontevedra y Galicia, y se cita también por primera vez para la provincia de A Coruña a Porcellio herminiensis.

De esta forma la fauna conocida de isópodos terrestres de Galicia esta formada por 38 especies, dos de ellas son endémicas de Galicia, Oniscus galicianus y Porcellionides rufocinctus. Pontevedra con 28 especies es la provincia más rica, seguida de A Coruña con 24 especies, Orense con 15 especies y Lugo con 14 especies (Tabla 1 ).

El grado de conocimiento de los isópodos gallegos es relativamente alto si se compara con el de otras provincias, y también su riqueza en especies es elevada, aunque por provincias queda por debajo de otras como por ejemplo Navarra (37 especies); Málaga (37 especies); Gerona (38 especies); Barcelona (43 especies) o las Islas Baleares, la más rica con 66 especies (datos de elaboración propia). Sin duda nuevos estudios permitirán ampliar la lista de los isópodos terrestres gallegos.

\section{Agradecimientos}

Quiero expresar mi agradecimiento a la Dra. Begoña Sánchez, conservadora del Museo Nacional de Ciencias Naturales de Madrid, por su ayuda para la consulta de la colección de isópodos del Museo. También quiero expresar mi agradecimiento a los revisores, ya que con sus comentarios han contribuido a mejorar el presente trabajo.

\section{Referencias}

Arcangeli, A., 1924. Contributo alla conoscenza degli isopodi della Catalogna. Trabajos del Museo de Ciencias naturales de Barcelona, 4: 3-29.

Arcangeli, A., 1925. Isopodi terrestri della Spagna settentrionale ed orientale, raccolti dal Dr. F. Haas negli anni 1914-1919. Abhandlungen der senckenbergischen naturforschenden Gesellschaft, 39: 131-137.

Arcangeli, A., 1935. Isopodi terrestri di caverne della Spagna (Collezione del Museo di Storia naturale di Madrid). Eos, 10: 171-195.

Arcangeli, A., 1936. Gli isopodi terrestri del Portogallo. Bollettino del Laboratorio di Zoologia generale e agraria della R. Scuola superiore d'Agricoltura in Portici, 29: 1-39.

Bellés, X., Damians, J. \& Pretus, J. L., 1989. «Minor-87»: Una campanya biospeleologica a Menorca. Endins, 14-15: 69-75.

Bilton, D., 1992. A new species of Oniscus Linnaeus, 1758 (Crustacea; Isopoda; Oniscidea) from northern Spain, with a revised key to members of the genus. Zoological Journal of the Linnean Society, 104: 117-125. https:// doi.org/10.1111/j.1096-3642.1992.tb00919.x

Bilton, D., 1997. The Oniscus asellus complex (Crustacea: Isopoda: Oniscidea) in the Iberian Peninsula with the description of a new species. Revue suisse de Zoologie, 104: 217-227. https://doi.org/10.5962/bhl.part.79998

Budde-Lund, G., 1885. Crustacea Isopoda terrestria per familias et genera et species descripta. Copenhagen. $319 \mathrm{pp}$.

Cifuentes, J., 1984. Isópodos terrestes (Crust. Oniscoidea) de Navarra. Boletín de la Asociación española de Entomología, 8: 233-252.

Cifuentes, J., 2018. Sobre algunos caracteres morfológicos en la identificación de las especies del género Porcellio Latreille, 1804: El papel de las sedas-escamas. (Crustacea: Isopoda, Porcellionidae). Boletín de la Asociación española de Entomología, 42(1-2): 71-91.

Cifuentes, J., 2019. Contribution à la connaissance des Trichoniscoides Sars, 1899 ibériques, avec la description de deux nouvelles espèces: Trichoniscoides marinae n. sp. et Tr. enoli n. sp. (Crustacea: Isopoda, Trichoniscidae). Boletín de la Asociación española de Entomología, 43(1-2): 27-54.

Cifuentes, J. \& Tinaut, A., 2018. Isópodos terrestres (Crustacea, Isopoda) de la cueva de Nerja (Málaga, España). Boletín de la Asociación española de Entomología, 42(1-2): 39-48.

Cifuentes, J. \& Tinaut, A., 2019. Isópodos terrestres (Crustacea, Isopoda, Oniscidea) de las cavidades del Parque Natural de la Sierra Norte de Sevilla (España). Boletín de la Asociación española de Entomología, 43(1-2): 79-90.

Coiffait, H., 1959. Enumération des grottes visitées 19501957 (neuvième série). Archives de Zoologie Expérimentale et Générale, 97: 209-465.

Cruz, A., 1989. Isópodos terrestres de Menorca (Crustacea, Isopoda, Oniscidea). Endins, 14-15: 89-93.

Cruz, A., 1991. Isópodos terrestres de la colección del Museu de Zoología de Barcelona (Crustacea, Oniscidea). Miscelánea zoológica, 15: 81-102.

Cruz, A., 1992. Los Halophilosciidae Verhoeff, 1908 de la Península Ibérica e Islas Baleares (Isopoda: Oniscidea). Boletín de la Asociación española de Entomología, 16: 113-121.

Cruz, A., 1993. El género Armadillidium Brandt, 1833 en la Península Ibérica y Baleares (Isopoda, Oniscidea, 
Armadillidiidae). Boletín de la Asociación española de Entomología, 17(1): 155-181.

Dalens, H., Rousset, A. \& Fournier, D., 1996. Les formes épigées du genre Oritoniscus (Crustacea, Isopoda, Oniscidea). I. Le complexe Oritoniscus flavus. Revue suisse de Zoologie, 103: 623-641. https://www.biodiversitylibrary.org/part/79964\#/summary

De Buen, O., 1887. Materiales para la fauna carcinológica de España. Anales de la Sociedad española de Historia Natural, 16: 405-434.

Dollfus, A., 1892. Catalogue raisonné des isopodes terrestres de l'Espagne. Anales de la Sociedad española de Historia natural, 21: 161-190.

Dollfus, A., 1893. Catalogue raisonné des isopodes terrestres de l'Espagne (1er supplément). Anales de la Sociedad española de Historia natural, 22: 47-51.

Dollfus, A., 1896. Les isopodes terrestres du nord de l'Afrique du Cap Blanc à Tripoli. Mémoires de la Société zoologique de France, 9: 523-553.

Dollfus, A., 1897. Tableau iconographique des Philoscia d'Europe. Feuille des jeunes Naturalistes, $3^{\text {ème }}$ Série, 27: 70-95.

Español, F., 1958. Contribución al conocimiento de los artrópodos y moluscos terrestres de las Islas Columbretes. Miscelánea zoologica, 1(1): 3-37.

Fidalgo, I. \& Herrera, L., 1980. Contribución al conocimiento de los Isópodos de Navarra (Crustacea: Isopoda): relación de especies con sus localidades. Boletín de la Real Sociedad Española de Historia Natural (Sección Biológica), 78: 311-315.

Galán, C., 2003. Ecología de la cueva de Guardetxe y del MSS circundante: un estudio comparado de ecosistemas subterráneos en materiales del Cretácico tardío del Arco Plegado Vasco. Sociedad de Ciencias Aranzadi. Disponible en: https://telesforo.aranzadi-zientziak.org/ karstologia/pdf-s/GUARDETXE.PDF

Galán, C., 2006. Fauna cavernícola y poblaciones bacteriales de la sima y río subterráneo de mondmilch de Alzola (Gipuzkoa). Sociedad de Ciencias Aranzadi. Disponible en: www.aranzadi.eus/fileadmin/docs/espeleologia/AlzolaBiolTr.pdf

Galán, C. \& Nieto, M., 2011. Fauna hipógea del mss y mesocavernas en conglomerados permo-triásicos (Mandoegi, Gipuzkoa). Sociedad de Ciencias Aranzadi. Disponible en: www.aranzadi.eus/.../fauna-hipogea-del-mss-y-mesocavernas-en-conglomerados-permo-triasicos-mandoegi-gipuzkoa

García, L., 2005. Primer registro de Armadillidium album Dollfus, 1887 (Isopoda: Crinocheta: Armadillidiidae) para Galicia y confirmación de la especie para el litoral atlántico ibérico. Boletín de la Sociedad Entomológica Aragonesa, 37: 310-311.

García, L., 2008. Els isòpodes terrestres (Crustacea: Isopoda: Oniscidea) del Parc Natural de l'illa de sa Dragonera (Illes Balears, Mediterrània occidental). Bolletí de la Societat d'Història Natural de les Balears, 51: 203-224.

García, L., 2009. Les “someretes” (Crustacis isòpodes terrestres: Isopoda, Oniscidea) de la vall de Sóller (Mallorca). Aproximació a una biodiversitat menystinguda. In: III
Jornades Estudis Locals a Sóller. Ajuntament de Sóller: 129-146.

García, L., 2013. Isópodos terrestres (Crustacea: Oniscidea) recolectados en cavidades subterráneas de Jaén. In: Los invertebrados de hábitats subterráneos de Jaén. Pérez Fernández, T. y Pérez Ruiz, A. (Coord.). Grupo de Espeleología de Villacarrillo: 78-87.

García, L. \& Cruz, A., 1993. Els isopòdes terrestres (Crustacea: Isopoda: Oniscidea). In: Història natural de l'Arxipèlag de Cabrera. Alcover, J., Ballesteros, E. \& Fornos, J. (Eds.). Monografies de la Societat d'Historia natural de les Balears, 2: 323-332.

García, L. \& Cruz, A., 1996. Els isopòdos terrestres (Crustacea: Isopoda: Oniscidea) de les Illes Balears: catàleg d'espècies. Bolletí de la Societat d'Història natural de les Balears, 39: 77-99.

García, L. \& Pérez Fernández, A., 2017. Eluma caelata (Miers, 1877) un isópodo terrestre (Crustacea: Oniscidea: Armadillidiidae) recolectado en las cuevas de Navilla de Fuente Acero (Cazorla, Jaén) y sus registros en el medio subterráneo andaluz. Monografías Bioespeleológicas, 12: 14-18.

García, L., Gross, A. \& Riddiford, N., 2003. Armadillidium album, un isòpode terrestre nou per a la fauna balear (Isopoda, Crinocheta, Armadillidiidae). Bolleti de la Societat d'Història natural de les Balears, 46: 91-94.

González Silvestre, J. V., 2015. Memorias del inframundo: Bioespeleología I. Gota a gota, 7: 1-13

Gregory, S., Lee, P., Read, H. J. \& Richards, P., 2012. Woodlice (Isopoda: Oniscidea) collected from northwest Spain and northern Portugal in 2004 by the british myriapod and isopod group. Bulletin of the British Myriapod \& Isopod Group, 26: 6-23.

Jackson, H., 1926. Woodlice from Spain and Portugal, with an account of Benthana, a sub-genus of Philoscia. Proceedings of the zoological Society of London, 96(1): 183-201. https://doi.org/10.1111/j.1096-3642.1926. tb01541.x

Jeannel, R. \& Racovitza, E. G., 1918. Énumération des grottes visitées, 1913-1917 (sixième série). Biospeologica, 39. Archives de Zoologie Expérimentale et Générale, 57: 203-470.

Jeannel, R. \& Racovitza, E. G., 1929. Énumération des grottes visitées, 1918-1927 (septième série). Biospeologica, LIV. Archives de Zoologie Expérimentale et Générale, 68: 293-608, pl. II.

Koch, C., 1856. Crustacea. In: Die Thiere Andalusiens nach dem Resultate einer Reise zusammengestelt, nebst dem Beschreibung von 249 neuen oder bis jetzt noch umbeschrieben Gattungen und Arten. W. G. Rosenhauer. Theodor Blaesing. Erlangen: 418-423.

Pablos, F., 1964. Isópodos de las islas Medas. Publicaciones del Instituto de Biología Aplicada, 36: 97-100.

Pablos, F., 1966. Sobre la presencia de Oniscus asellus var. jaccetanus, en el Pirineo aragonés. Publicaciones del Instituto de Biología Aplicada, 41: 109-112.

Pollo Zorita, A. M., 1982. Estudio de algunos oniscoides (Isopoda Oniscidae) de la vertiente leonesa de la Cordillera Cantabrica. Boletín de la Asociación española de Entomología, 6: 49-55. 
Pollo Zorita, A. M., 1986a. Oníscidos de la cuenca alta del río Tajo (Isopoda, Crustacea). Graellsia, 41: 173-189.

Pollo Zorita, A. M., 1986b. Oníscidos en suelos de bosques quemados de la zona del alto Tietar (Ávila) (Isopoda, Crustacea). Graellsia, 41: 191-196.

Preudhomme De Borre, A., 1886. Crustacés Isopodes recuillis par feu Camille Van Volxen pendant son voyage en Portugal, en 1871. Bulletin de la Société entomologique de Belgique, 30(Bull. C.R. Séances): CXII-CXIII.

Racovitza, E., 1907. Biospéologica. IV. Isopodes terrestres (première série). Archives de Zoologie expérimentale et générale, 4ème Série, 7: 145-225.

Reboleira, A. S. P. S., Goncalves, F., Oromí, P. \& Taiti, S., 2015. The cavernicolous Oniscidea (Crustacea: Isopoda) of Portugal. European Journal of Taxonomy, 161: 1-61. https://doi.org/10.5852/ejt.2015.161

Schmölzer, K., 1955a. Isopoda terrarum mediterranearum. 1. Mitteilung: Über neue und bekannte Landasseln der Pyrenaenhalbinsel. Eos, 31: 155-215.

Schmölzer, K., 1955b. Landasseln aus Spanien, gesammelt von Prof. Dr. Ing. H. Franz. Eos, 31: 311-321.

Schmölzer, K., 1971. Die Landisopoden der Iberischen Halbinsel. Monografias de Ciencia moderna, $\mathrm{n}^{\circ} 80$. Madrid. XI + 161 pp.

Soika, A. G., 1954. Ecologia, sistematica, biogeografia ed evoluzione del Tylos laterillei Auct. (Isop. Tylidae). Bollettino del Museo Civico di Storia Naturale di Venezia, 7: 63-83.

Schmalfuss, H., 2003. World catalog of terrestrial isopods (Isopoda: Oniscidea). Stuttgarter Beiträge zur Naturkunde A (Biologie), 654: 1-341.

Tabacaru, I., 1974. Espèces du Trichoniscus (Crustacea, Isopoda) de l'île de Majorque. Travaux de l'Institut de Spéologie “Emile Racovitza”, 13: 213-221.

Taiti, S. \& Rossano, C., 2015. Terrestrial isopods from the Oued Laou basin, north-eastern Morocco (Crustacea: Oniscidea), with descriptions of two new genera and seven new species. Journal of Natural History, 49(33-34): 2067-2138. https://doi.org/10.1080/002229 33.2015 .1009512

Vadell, M., 2003. Fauna invertebrada de las cavidades del barranc de Sa Coma del Mal Pas (Calvià i Palma, Mallorca). Endins, 25: 107-116.

Vadell, M. \& Zaragoza, J. A., 2005. Estudio preliminar de la fauna invertebrada terreste de la cova des Coll (Felanitx, Mallorca). Endins, 27: 187-204.

Vadell, M., Zaragoza, J. A., Jordana, R., García, L., Gràcia, F. \& Clamor, B., 2006. Nuevas aportaciones al conocimiento de la fauna cavernícola terrestre de las coves del Pirata, cova des Pont, cova de sa Piqueta y la cova des Xots (Manacor, Mallorca, Baleares). Endins, 29: 75-98.
Vandel, A., 1946. Crustacés isopodes terrestres (Oniscoïdea) épigés et cavernicoles du Portugal. Anaïs da Faculdade de Ciências do Porto, 30: 135-427.

Vandel, A., 1951. Le genre Porcellio (Crustacés; Isopodes: Oniscoidea). Évolution et systématique. Mémoires $d u$ Muséum national d'Histoire naturelle, Série A, Zoologie, 3: 81-192.

Vandel, A., 1952. Sur les caractères de Porcellio nigrogranulatus Dollfus 1892 (isopode terrestre). Bulletin du Muséum national d'Histoire naturelle, $2^{\text {ème }}$ Série, 24: 522-525.

Vandel, A., 1953. Les isopodes terrestres des provinces d'Almería et de Granada. Archivos del Instituto de Aclimatación, 1: 45-75.

Vandel, A., 1954. Étude des isopodes terrestres recueillis aux Îles Canaries par J. Mateu en mars-avril 1952. Mémoires du Muséum national d'Histoire naturelle, Série A, Zoologie, 8: 1-60.

Vandel, A., 1957. Nouvelles espèces et sous-espèces appartenant au genre Oritoniscus (Isopodes terrestres). Bulletin de la Société d'Histoire naturelle de Toulouse, 92: 7-16.

Vandel, A., 1958. Les porcellions catalans et l'origine des porcellions français (Crustacés, Isopodes terrestres). Revue française d'Entomologie, 25: 129-148.

Vandel, A., 1960. Faune de France, vol. 64. Isopodes terrestres (première partie). Paris. 416 pp.

Vandel, A., 1961. Les isopodes terrestres de l'Île de Minorque. Archives de Zoologie expérimentale et générale, 4e Série, 99: 249-265.

Vandel, A., 1962. Faune de France, 66. Isopodes terrestres (deuxième partie). Paris. pp. 417-931.

Vázquez Felechosa, M. \& Anadón, A., 2001. Las familias Oniscidae Latreille, 1758 y Armadillidiidae Brandt, 1804 (Isopoda, Oniscidea) de la colección de artrópodos de la Universidad de Oviedo (Asturias). Boletín Ciencias Naturales IDEA, 47: 53-77.

Vázquez Felechosa, M., Anadón, A., Rosa-García, R., Ocharan, F. J., Melero, V. X., Monteserín, S. \& R. Ocharan, S., 2004. La fauna de Isópodos terrestres (Isopoda, Oniscidea) de la Reserva de la Biosfera de Muniellos (Asturias, norte de España). Boletín de la Asociación española de Entomología, 28(3-4): 11-23.

Verhoeff, K., 1901. Über paläarktische Isopoden (7. Aufsatz). Zoologischer Anzeiger, 24: 403-408, 417-421.

Vivar, J., De La Vega, I. \& Cifuentes, J., 1984. Aportaciones al conocimiento de los isópodos terrestres del País Vasco, La Rioja y provincias limítrofes. Instituto de Estudios Riojanos Berceo. Zubia, 2: 23-37. https://dialnet.unirioja.es/servlet/articulo? codigo $=110163$ 